\title{
Spectral energy distributions of quasars selected in the mid-infrared
}

\author{
M. Lacy $^{1}$, A. Sajina ${ }^{2}$, A. O. Petric ${ }^{3}$, S. E. Ridgway ${ }^{4}$, D. M. Nielsen ${ }^{5}$, \\ T. Urrutia ${ }^{6}$, D. Farrah ${ }^{7}$, and E. L. Gates ${ }^{8}$ \\ ${ }^{1}$ National Radio Astronomy Observatory, 520 Edgemont Road, Charlottesville, VA22903, USA \\ ${ }^{2}$ Dept. of Physics and Astronomy, Tufts University, Medford, MA02155 \\ ${ }^{3}$ Department of Astronomy, California Institute of Technology, 1200 E. California Boulevard, \\ Pasadena, CA91125, USA \\ ${ }^{4}$ National Optical Astronomy Observatory, 950 N. Cherry Avenue, Tucson, AZ85719, USA \\ ${ }^{5}$ Dept. of Astronomy, University of Wisconsin, Madison, WI53706 \\ ${ }^{6}$ Liebnitz Institut für Astrophysik Astrophysics Potsdam, An der Sternwarte 16, 14482, \\ Potsdam, Germany \\ ${ }^{7}$ Department of Physics and Astronomy, University of Sussex, Falmer, Brighton BN1 9QH, UK \\ ${ }^{8}$ UCO/Lick Observatory, University of California, 1156 High Street, Santa Cruz, CA95064 \\ email: mlacy@nrao.edu
}

\begin{abstract}
We present preliminary results on fitting of SEDs to $142 z>1$ quasars selected in the mid-infrared. Our quasar selection finds objects ranging in extinction from highly obscured, type-2 quasars, through more lightly reddened type-1 quasars and normal type-1s. We find a weak tendency for the objects with the highest far-infrared emission to be obscured quasars, but no bulk systematic offset between the far-infrared properties of dusty and normal quasars as might be expected in the most naive evolutionary schemes. The hosts of the type-2 quasars have stellar masses comparable to those of radio galaxies at similar redshifts. Many of the type-1s, and possibly a one of the type-2s require a very hot dust component in addition to the normal torus emission.
\end{abstract}

Keywords. galaxies:active, quasars: general, infrared: galaxies

\section{Introduction}

Over the past three years we have been conducting an extensive campaign of follow-up spectroscopy of dust obscured quasars, using the selection technique described in Lacy et al. $(2004 ; 2007)$. We now have over 700 spectra of AGN and quasars selected in this manner, forming the Spitzer Mid-InfraRed Quasar Survey (SMIRQS). SED fitting has been a key activity carried out in parallel with the spectroscopic follow-up. It allows us to separate the stellar, AGN and starburst components of the quasar and its host, it enables a consistency check on the spectroscopic redshift (which may be of low signal-to-noise in the case of highly-obscured objects), it helps with classification of $z>1$ objects between type-2 and reddened type-2 that lack rest-frame optical (i.e. near-infrared) spectra, and it allows us to test theories for the origin and nature of dusty quasars and their various SED components.

The two most popular explanations for the existance of dust-reddened quasars are orientation, where a dusty nuclear torus provides a large amount $\left(A_{V}>10\right)$ of extinction to the quasar (Antonucci 1993), and evolution, where a quasar begins its life highly obscured (again $A_{V}>>10$ ) following a galaxy merger and ensuing starburst, but then gradually expels its dusty envelope and eventually shines as an unobscured quasar 

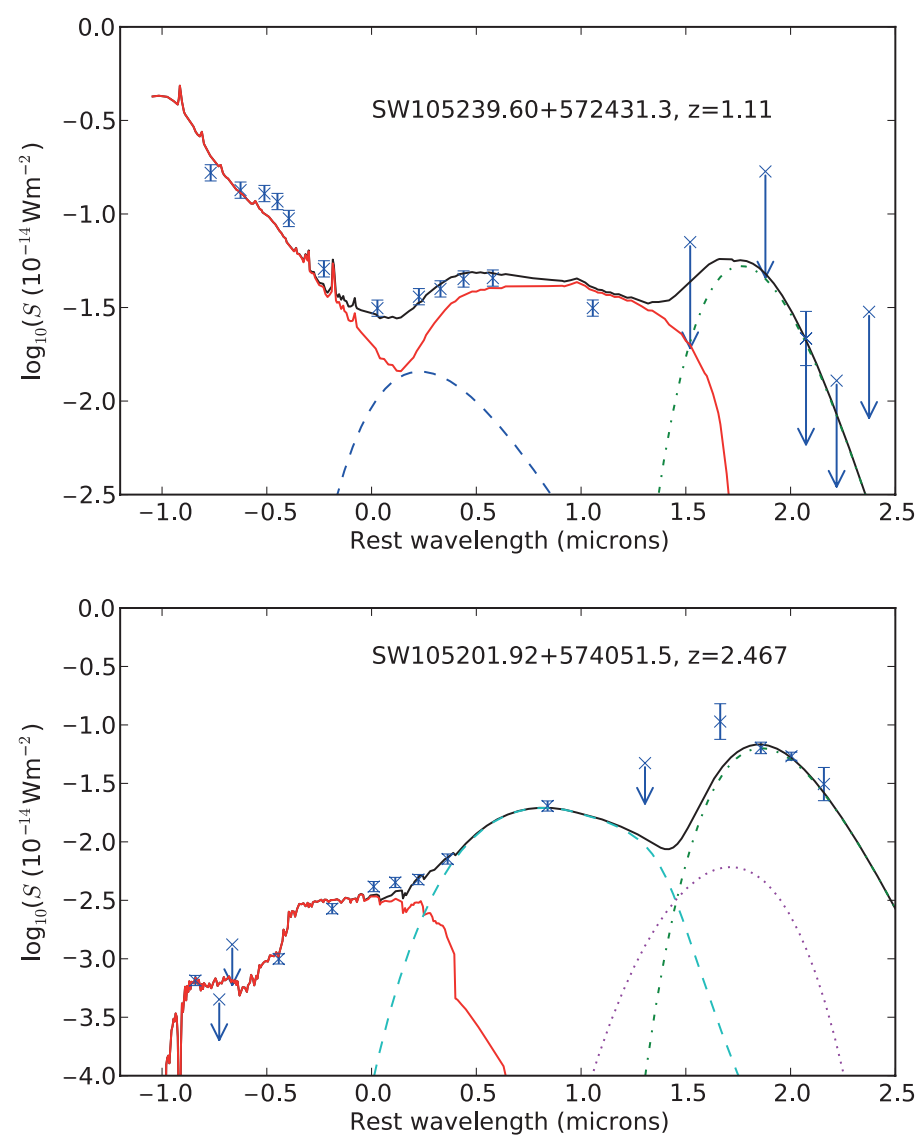

Figure 1. Example SED fits: Top A type-1 quasar SED fit, split into components (from left to right), an optical/IR quasar template (solid), a hot dust bump (dashed line) and a far-infrared modified black body (dot-dashed line). Bottom a type-2 quasar fit, components (from left to right), stellar light (solid), AGN-heated dust (dashed line), a warm dust component (dotted line) and a far-infrared modified black body (dot-dashed line).

(Sanders et al. 1988; Hopkins et al. 2008). The relative roles of orientation and evolution have been a topic of some debate, one simple, if somewhat naive, test is whether the obscured quasars show significantly more signs of ongoing star formation than their unobscured counterparts, consistent with them being at an earlier stage in the evolutionary sequence. We have also used our SED fits to study the very hot dust component in the near-infrared. The strength of this recently-recognized component appears to correlate with the bolometric luminosity of the quasar. Mor \& Trakhtenbrot (2011) use WISE data to demonstrate that, because this dust is above the sublimation temperature of silicate grains, it must arise in pure graphite grains in clouds beyond the dust-free broad-line region, but inside the torus.

In this paper we present the results of a preliminary analysis of the $142 z>1$ objects in our mid-infrared selected quasar sample (two example fits are shown in Figure 1). Full results will be presented in Farrah et al. (in preparation) and Petric et al. (in preparation). 


\section{The very hot component}

We confirm the need for a very hot dust component in the near-infrared spectra of many of our type- 1 objects, and also marginal evidence in one of our type-2s. Extinction is probably responsible for the lack of these in most of our dusty type- 1 and type- 2 quasars. The apparent need for a very hot component in one of our type-2s (SW105213.39+571605.0; $z=1.242$ ) is intriguing, and may help to constrain the geometry of the emission from this component.

\section{Stellar masses}

The objects classified as type-2 have had their stellar masses estimated by fitting stellar population models from Maraston (2005). We fit models that are very simple single or dual stellar populations (see Lacy et al. 2011 for details). The results (Figure 2, left) show that the mean stellar masses are around $2 \times 10^{11} M_{\odot}$, but with a wide range, $10^{10-12.4} M_{\odot}$. There is a loose correlation with quasar luminosity. These stellar masses are similar to those seen in radio galaxies (de Breuck et al. 2010) at these redshifts, somewhat surprising given that Kukula et al. (2001) claim a significant difference in the host galaxy luminosities of radio-loud and radio quiet quasars at these redshifts.

\section{The cool dust component}

Over $50 \%$ of our quasars show a cool dust component, visible as detections in archival Herschel data and/or Spitzer data. The detected objects have far-infrared luminosities characteristic of Ultraluminous Infrared Galaxies (ULIRGS). Dust temperatures are seen to vary widely, from $\sim 30$ to $>100 \mathrm{~K}$. We suspect many of the warmer objects may have contributions to their dust heating from AGN (see also Schumacher et al. 2012).

The luminosity of the cold component correlates with the AGN luminosity (Figure 2, right) with a logarithmic slope well below unity, as also seen in low-z, normal quasars
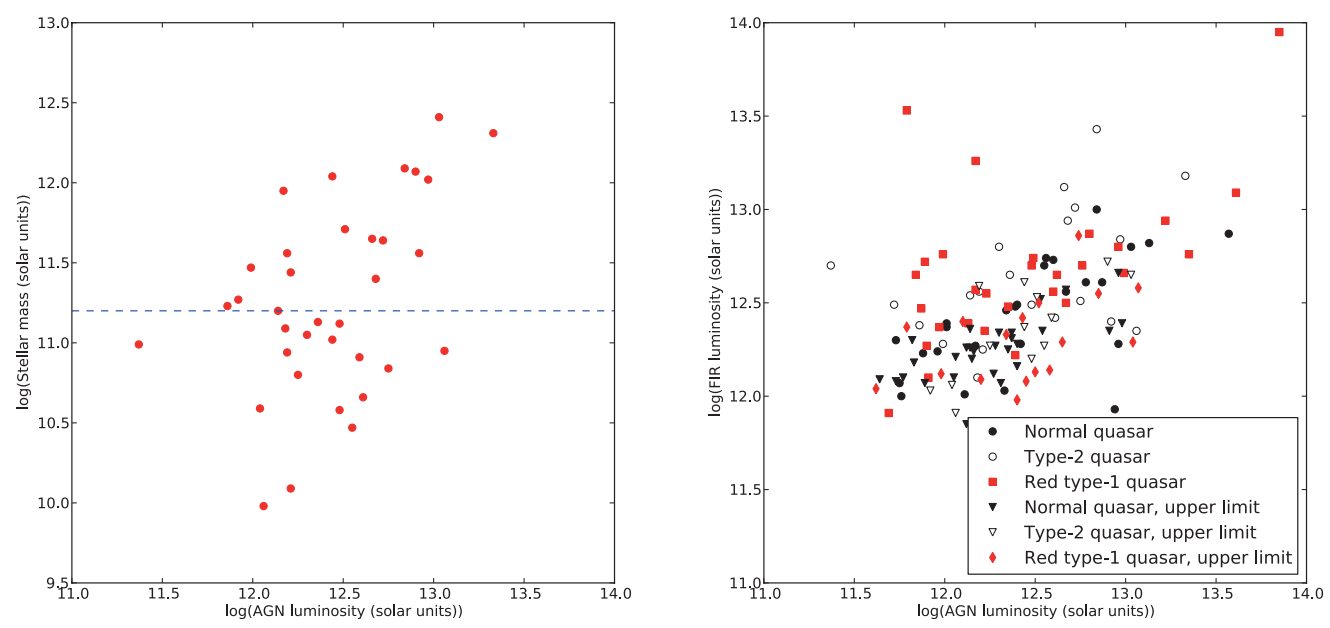

Figure 2. Left Stellar masses of the $z>1$ type-2 quasars in the sample. The dashed horizontal line shows the mean stellar mass of both this sample and the radio galaxy sample of de Breuck et al. (2010). Right Far-infrared luminosity versus AGN luminosity for our quasars. 
e.g. Netzer et al. (2007). For the most part, the dusty type-1 and type-2 quasars fall on the same correlation as the normal type-1s, however, all the objects well above the correlation are dusty or type-2 objects.

\section{Discussion}

Our results show that more than half of mid-infrared selected quasars, regardless of optical classification as type-1, reddened type- 1 or type- 2 , are hosted by galaxies having the far-infrared luminosities of ULIRGs, although the amount of far-infrared emission contributed by the AGN is unclear in many cases. The most far-infrared luminous of these quasars are all obscured to some extent, however, thus evolutionary schemes, in which dusty quasars are an early stage in the evolution of quasars are certainly not ruled out by this study.

The stellar masses of the host galaxies of the type-2 quasars are high, consistent with those of radio galaxies at the same epoch, suggesting that these quasars are relatively mature systems even at $z \sim 2$. Where we have been able to estimate CO masses, these seem lower than submm-selected galaxies at these redshifts, also consistent with the idea that these are relatively mature systems (Lacy et al. 2011).

\section{References}

Antonucci, R. 1993, ARAEA A, 31, 473

de Breuck, C. et al. 2010, ApJ, 725, 36

Hopkins, P. F., Hernquist, L., Cox, T. J., \& Keres, D. 2008, ApJS, 175, 356

Kukula, M. J., Dunlop, J. S., McLure, R. J., Miller, L., Percival, W. J., Baum, S. A., \& O’Dea, C. P. 2001, MNRAS, 326, 1533

Lacy, M. et al. 2004, ApJS, 154, 166

Lacy, M. Petric A. O., Sajina, A., Canalizo, G., Storrie-Lombardi, L. J., Armus, L., Fadda, D., \& Marleau, F. R. 2007, AJ, 133, 186

Lacy, M., Petric A. O., Martínez-Sansigre, A., Ridgway, S. E., Sajina, A. Urrutia, T., \& Farrah, D. 2011, $A J, 142,196$

Maraston, C. 2005, MNRAS, 362, 799

Mor, R. \& Trakhtenbrot, B. 2011, ApJ, 737, L36

Netzer, H. et al. 2007, ApJ, 666, 806

Sanders, D. B., Soifer, B. T., Elias, J. H., Madore, B. F., Matthews, K., Neugebauer, G., \& Scoville, N. Z. 1988, ApJ, 328, L35

Schumacher, H. et al. 2012, MNRAS, submitted 